\title{
Liderazgo resiliente ante escenarios prospectivos
}

\author{
Afonso Finol, María del Pino* \\ *Universidad Privada “Dr. Rafael Belloso Chacín” URBE, \\ Maracaibo, Venezuela \\ Correo autor principal: mpaf12@gmail.com
}

Recibido: 15 mayo de 2019 Aprobado: 20 junio de 2019

\begin{abstract}
Resumen
El objetivo de este artículo es describir la resiliencia y presentar una reflexión acerca del reto que tienen los líderes resilientes, sobre todo en el contexto de una gerencia prospectiva, logrando plantear desde la perspectiva teórica diversos aportes en esta materia, el cual contribuye a fortalecer su accionar en las organizaciones con respecto a la manera en la que gerentes y colaboradores tengan la facilidad de adaptarse a esas situaciones y oportunidades futuribles. Se estructuró bajo un enfoque de tipo documental, analítico, sustentado en los trabajos de Robbins (2015), Meneghel, Salanova y Martínez (2013), Trujillo (2011), entre otros. Se refiere a la necesidad de que la gerencia asuma un nuevo compromiso ante una realidad cambiante, por ello, se concluye que el liderazgo resiliente estimula a las demás personas para que puedan enfrentarse efectivamente a las adversidades, superando los obstáculos, y generando un mayor crecimiento tanto personal como profesional, es así como el líder debe disponer de una serie de competencias que serán claves en su accionar ante escenarios prospectivos.
\end{abstract}

Palabras clave: Liderazgo, Resiliencia, Prospectiva, Escenarios. 


\title{
Resilient leadership in prospective scenarios
}

\begin{abstract}
The objective of this article is to describe the resilience and present a reflection about the challenge that resilient leaders have, especially in the context of a prospective management, being able to raise from the theoretical perspective diverse contributions in this matter, which contributes to strengthen their to act in organizations with respect to the way in which managers and collaborators have the facility to adapt to those situations and future opportunities. It was structured under a documentary, analytical approach, based on the papers of Robbins (2015), Meneghel, Salanova y Martinez (2013), Trujillo (2011), among others. It refers to the need for management to assume a new commitment to a changing reality, therefore, it is concluded that resilient leadership encourages other people to be able to effectively face adversity, overcoming obstacles, and generating greater growth both personal and professional, this is how the leader must have a series of skills that will be key in his actions in prospective scenarios.
\end{abstract}

Key words: Leadership, Resilience, Prospective, Scenarios.

\section{Introducción}

El gran reto de la globalización ha de ser propiciar espacios dentro de un entorno competitivos, para lograrlo la gerencia no puede diseñar sus acciones en el día a día, debe considerar y evaluar un sinnúmero de variables que intervienen en el entorno organizacional, desde una revisión del pasado, comparada con el presente, que permite tener una visión de la posibilidades que podrían presentarse en un futuro inmediato y hasta uno no tan cercano.

En este orden de ideas, la gerencia debe tener la capacidad de visualizar una dinámica de posibles escenarios económicos futuros o futuribles, no se trata de adivinar el futuro, sino más bien a adelantarse a las posibles situaciones que pudieran presentarse, con el fin de aprovechar las fortalezas y oportunidades que se le presentan a la organización.

De lo antes indicado, resulta interesante preguntarse qué papel juega el líder o debe asumir ante este panorama, basado en que la actitud de gran parte de colaboradores y gerentes descansa todavía en el paradigma de la transmisión de información, en esquemas tradicionales de hacer las cosas por costumbre y en algunos casos bajo un estilo clásico y conservador. En tal sentido, llama la atención observar con mucha frecuencia algunos gerentes que prefieren mantenerse apegados a sus viejos esquemas, en lugar de facilitar momentos para el pensamiento crítico, la reflexión y el pensamiento prospectivo, así como el evaluar la importancia de adelantarse y prepararse a las diversas opciones de escenarios futuribles, para la consolidación de mejores acciones para el logro de sus objetivos y enfrentar los retos plateados en el marco de la globalización.

Por ello, la gestión y planificación basada en las estrategias prospectivas, requiere de un liderazgo que permita orientar al personal en la búsqueda de los mejores escenarios u opciones y el compromiso de su actitud al accionar, sobreponiéndose y adaptándose a la diversidad de situaciones que en un sistema globalizado se presentan, logrando una excelencia en los servicios prestados, generando así un mayor compromiso hacia el logro de los objetivos planteados.

Por tanto, el liderazgo y la resiliencia han sido objeto de estudio en diversos contextos, 
sin embargo, la combinación de ambas variables implica la capacidad de una persona de recuperarse, adaptándose con éxito a las situaciones o adversidades enfrentadas, logrando desarrollar su entorno mediato e inmediato dentro de un contexto que fomenta la resilencia de todas las personas que se encuentran a su alrededor, convirtiendo sus pensamientos, emociones, conversaciones en una herramienta de resiliencia que pueda proporcionar la misma energía a su entorno para que puedan estar más fortalecidos.

Es así como un liderazgo resiliente permite que las competencias del líder puedan ayudar a las demás personas en adaptarse o recuperarse de cualquier situación, y específicamente estar preparados para su accionar en los escenarios futuros probables, por ello, sus orientaciones deben ser consideradas como catalizadoras, inspirando a las personas en búsqueda de metas claras donde puedan lograrlas por sí solos o en trabajo de equipo, por tanto, un líder resiliente debe tener una visión proactiva, debe ser visionario, con capacidad para reconocer los peligros de las adversidades buscando mejores alternativas para enfrentarlas, así como disponer de capacidad e iniciativa para brindar su apoyo a las personas que se encuentran a su alrededor.

Si se realiza una revisión de las variables antes mencionadas pudiera emerger nuevos conceptos a nivel organizacional, como lo sería el liderazgo resiliente prospectivo, donde el líder como eje fundamental en el equilibrio de sus seguidores, sea capaz de fomentar su capacidad de superar las adversidades e ir más allá, en anticiparse a esos posibles eventos al punto de poder evitar que se conviertan en circunstancia que afecten a la organización de manera considerable.

\section{El lider y la resiliencia}

Dentro de las organizaciones, la resiliencia implica la capacidad de lograr un equilibrio mental y emocional en circunstancias adversas, afrontando mediante la perseverancia, toma de decisiones, autoconfianza, generando un ambiente positivo que estimule la integración de sus miembros en búsqueda de una equidad emocional compartida.

De acuerdo al planteamiento de Robbins (2015), el liderazgo implica la habilidad para influir en un grupo y dirigirlo hacia el logro de un objetivo o conjunto de metas, por tanto, la fuente de ésta influencia puede ser formal, como la que proporciona una jerarquía administrativa en una organización, sin embargo, se debe tener una perspectiva clara en cuanto a que no todos los líderes son gerentes y viceversa. Por tanto, el simple hecho de que una organización otorgue a sus gerentes ciertos derechos formales, no garantiza que sean capaces de dirigir con eficacia, ni lograr gestionar los escenarios prospectivos.

Sobre este particular, Richardson (2014), afirma que existen diversos protectores internos de un líder resiliente, los cuales se basan en: proporcionar un servicio a otros o a una causa en particular; aplicar estrategias de convivencia, mediante la asertividad, resolución de conflictos, control de impulsos; capacidad de socializar, ser un amigo confiable, crear vínculos afectivos; sentido del humor; control interno; autonomía; visión proactiva del personal; así como automotivación; confianza en sí mismo, 
los cuales serán un apoyo para su capacidad de dirigir a un grupo, así como el fomento de la confianza entre sus miembros.

Apoyando lo anterior, Salazar (2015), señala que dentro de todo proceso de resiliencia, el líder debe proporcionar a las personas que han enfrentado adversidades los siguientes elementos: (a) proporcionar afecto; (b) crear y transmitir expectativas elevadas; (c) brindar nuevas oportunidades.

Proporcionar afecto: es considerado un elemento determinante en apoyo a promover la resiliencia, por tanto, se ha evidenciado que es difícil superar la adversidad si no se proporciona un afecto a las personas que lo requieren, por tanto, el mismo no necesariamente debe provenir de familiares, amigos directos, puede ser de cualquier persona que tenga cierta capacidad de empatía hacia el otro.

Crear y transmitir expectativas elevadas: las mismas deben ser factibles o realistas para que sean motivadores efectivos, cuyo objetivo se vincula hacia el desarrollo de las habilidades y aprovechar el potencial del resiliente.

Brindar nuevas oportunidades: se vincula directamente a otorgar al resiliente nuevas experiencias y responsabilidades en asumir funciones y/o cargos, solucionar conflictos, crear estrategias, o lograr una influencia en otros, por tanto, esto permitirá a las personas que se encuentren en esta condición, ser parte o ejemplo de poder seguir sus actividades, es decir, ser un resiliente efectivo.

Por ello, Trujillo (2011), señala que el liderazgo resiliente implica un nuevo paradigma en el perfil del líder, justifica para la organización la capacidad para superar la adversidad proyectando consecuentemente respuestas $\mathrm{y}$ comportamientos de superación para afrontar las dificultades sobrevenidas. Es así como el mismo cobra relevancia en todas las instituciones, apoyando a su personal en la búsqueda de un equilibrio socioafectivo, que permita seguir siendo una clave para la efectividad de la organización, donde pueda aprovechar sus competencias, mejorando su calidad de vida y ser el principal motor del éxito en la sociedad. El liderazgo resiliente implica el tener el valor de actuar, deseo de asumir retos por decisiones tomadas de manera independiente y la habilidad de establecer confianza, fidelidad mediante un compromiso integral.

En consecuencia, el éxito organizacional, es imposible de alcanzar sin la excelencia individual de cada integrante de la organización, especialmente en puestos gerenciales la cual demanda más que competencia técnica, un verdadero liderazgo, que lo capacite para movilizar grupos de personas y lograr importantes objetivos a pesar de los obstáculos que comúnmente se evidencian en las organizaciones altamente exitosas.

Se infiere del planteamiento, que los gerentes además de planificar, organizar, ejecutar y evaluar las actividades de una organización, el papel primario de un gerente es influir en los demás para alcanzar con entusiasmo los objetivos establecidos. Tal situación requiere una persona muy motivada y con gran confianza en sí misma que le empujan a adquirir y utilizar el poder para lograrlas por medio de otras personas. 
Por lo antes planteado, el compromiso del líder en las organizaciones, es la capacidad para orientar a su personal de manera efectiva. En el siglo XXI el capital intelectual de una empresa, influye en el éxito de la gestión organizaciones. Por ello, los líderes competentes también establecen el contexto para que los grupos de interés puedan desempeñarse al máximo de eficiencia. La clave radica en la capacidad para administrar las operaciones con efectividad y sostener un alto desempeño a lo largo del tiempo, manteniendo un personal exitoso.

Dentro de este contexto, los retos y oportunidades del líder del siglo XXI en las organizaciones es proporcionarle un apoyo a la gerencia para el manejo de las relaciones laborales, así como en el fortalecimiento de su cultura, creando un entorno confiable, donde se labore sobre la base de criterios éticos que permitan establecer un clima ajustado a las necesidades de su talento humano. Como lo afirma Romero (2014), toda organización puede generar un valor agregado a todo el entorno, debido a que se logra descentralizar las funciones, asigna responsabilidades directas y especificas a otras áreas, así como un soporte a la gerencia del talento humano.

Por tanto, son variados los factores que influyen para lograr la calidad y el éxito organizacional, la actitud o el accionar del talento humano puede ser negativa o problemática si estas son incongruentes con los principios o valores de la organización; o positiva si es congruente con estos fundamentos, no presentando problemas. Sin embargo, tales posiciones pueden verse afectadas en su interior por el clima organizacional en que se encuentran insertos. Sobre la base de lo planteado, afirma Medina (2015)

El ejercicio del líder supone adaptar las operaciones a las capacidades y la motivación de las personas, potencializando su autoestima y su propio reconocimiento de sus capacidades para así forjar un grupo resiliente. Esto conduce a reconocer la importancia de conocer al otro para generar un contexto de crecimiento reciproco. Este proceso de creer en las capacidades ajenas significa ejercitar la confianza en los demás individuos, porque se aprende de todo, entender al grupo resiliente, permite reconocer las imperfecciones y da la oportunidad de generar pensamiento crítico frente a cada uno de ellos. De esta manera se crea un equipo en donde cada uno de sus integrantes es fuerte, se reconoce a sí mismo y puede confiar en los demás y eso es proporcionado por un eje central, el líder. (p.42).

Por tanto, el líder resiliente debe establecer los parámetros dentro de los cuales guiará a sus seguidores o les proporcionará la ayuda necesaria para convertirlos en nuevos guía resilientes, sin embargo, es un proceso complejo que requiere organización, dirigiendo sus esfuerzos hacia una meta en común, por ello, requiere de un proceso de aprendizaje colectivo, estimulando la comunicación, confianza, empatía y relaciones interpersonales claras y efectivas.

Por ello, las organizaciones de éxito consideran que sus empleados son activos que deben ser orientados de forma consciente $\mathrm{y}$ acorde con sus necesidades, por tanto, mientas las empresas más exitosas del futuro están trabajando desde hoy para asegurarse que en el futuro, tendrán trabajadores que estén deseosos de atacar los retos de la competencia 
y sean capaces de hacerlo bien. Esto significa, cada vez más, que deben tener capacidad para atraer a talentos superiores y estimular a los colaboradores de modo que su desempeño llegue a niveles máximos, por ello, dentro de este planteamiento, es pertinente que los líderes resilientes consideren las siguientes estrategias:

Diseñar puestos que tengan gran potencial para motivar. Los cargos de trabajo diseñados de modo que cumplan con los principios de la teoría de las características de los mismos, suelen ser más gratificantes que otros. A fin de determinar si los oficios deben ser rediseñados o no, los administradores deben evaluar la medida en que los empleados experimentan su trabajo como algo que tiene sentido, sienten una responsabilidad personal en los resultados de su trabajo y reciben retroalimentación adecuada.

\section{Proporcionar} retroalimentación constructiva con frecuencia. Cuando los empleados tienen un buen desempeño, decirlo les alienta a seguir adelante. Cuando tienen un mal desempeño, decírselos sugiere que deben considerar otro enfoque para la tarea o intensificar sus esfuerzos. Sin embargo, proporcionar retroalimentación correcta puede ser muy difícil. Una regla general sería que la retroalimentación se debe concentrar en el desempeño de las tareas y que se debe proporcionar de inmediato; además, no hay que criticar las características personales que los empleados difícilmente podrán cambiar.

Entregar recompensas por los comportamientos y los resultados deseados. De una forma u otra, el ser humano desea ser apreciado y que sus acciones sean valoradas, en este sentido, los empleados tienden a repetir los comportamientos que son valorados $y$ recompensados, luchando por alcanzar las metas que van ligadas a una acción motivadora. De igual forma, se evitan las actuaciones que pudieran acarrear castigos o limitar reconocimientos, en este sentido, los líderes efectivos se aseguran de que los premios y las sanciones, que experimentan los trabajadores estén alineados con las metas u objetivos de la organización.

Entregar recompensas que valoren los empleados. Para que las retribuciones sean motivadorasdebenreflejarcosasquelos empleados aprecien. Aquellas que quieren obtener se pueden determinar con sólo preguntarles. Algunos valoran los reconocimientos monetarios más que cualquier otra cosa, mientras que otros valoran la flexibilidad de horarios, la posibilidad de trabajar en proyectos especiales, las oportunidades de capacitación y desarrollo. En la medida de lo posible, los líderes efectivos encuentran la manera de utilizar diversas gratificaciones para motivar a distintos empleados.

\section{Competencias del nuevo líder resiliente}

El rol que ejerce el líder resiliente es fundamental en la vida de cada una de las personas que logra influir, sobre todo por la capacidad de aceptación que tiene ante los demás, por ello, su principal objetivo es lograr que cada resiliente posea una persona de confianza que lo estimule a seguir adelante, que lo apoye de manera directa e incondicional en cada etapa de su vida, el mismo, puede ser un familiar, amigo o compañero de trabajo, situación que permitirá que sea un ejemplo tanto a nivel personal como laboral, estimulando a otras personas en alcanzar sus metas. 
Señala Robbins (2015), que los lideres resilientes saben quiénes son, saben que es lo que creen y valoran; actúan de manera abierta, franca; a partir de esos valores y creencias. Sus seguidores los consideran personas éticas y confiables, por tanto, su principal cualidad es la confianza, comparten información, estimula una comunicación abierta, se apegan a sus ideales, por ello, llegan a tener fe en ellos. Sobre este particular, el autor citado, señala diversas competencias que caracterizar a un líder resiliente:

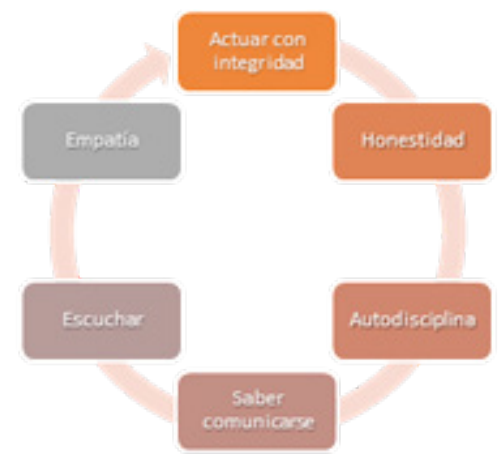

Figura 1. Competencias del líder resiliente. Robbins (2015), adaptado por Afonso (2017).

Actuar con integridad: Implica la capacidad del líder para realizar las cosas de manera correcta, siendo transparente en su ejercicio, cumplir con cada de las promesas planteadas, y sobre todo ser el verdadero guía que sus seguidores requieren.

Honestidad: Cualidad humana que debe poseer toda persona, en este caso los lideres resilientes, el cual implica en comportarse y expresarse con coherencia, de acuerdo a la verdad y a la justicia.

Autodisciplina: Todo líder debe estar en capacidad de aceptar y asumir sus responsabilidades, logrando que su comportamiento incida de manera positiva y no negativa en las personas que lo siguen, siguiendo adelante, alcanzando metas.

Saber comunicarse: Expresar sus ideas de manera clara, directa, logrando una retroalimentación de lo que se informa, donde todos puedan entender y tener la capacidad de opinar sin censura.

Escuchar: Existe un pensamiento claro planteado por Aristóteles, quien señalaba que antes que una persona logre persuadir a otra mediante la lógica, debe escuchar dos cosas importantes, su punto de vista y su dolor, por ello, antes de persuadir a alguien se debe escuchar, razón por la cual incrementará su credibilidad ante los demás.

Empatía: En momentos de crisis, sentir la preocupación o dolor ajeno como parte de sí, es un elemento generador de confianza, por ello, el líder resiliente debe estar en capacidad de ser reciproco con sus seguidores.

Lo anterior requiere un proceso de dirección que permita a los individuos desarrollar sus capacidades en búsqueda de un equilibrio personal o profesional, logrando sacarle provecho a su potencial, convirtiendo al talento humano en verdadera ventaja competitiva, para ello, es necesario apoyarlos en el desarrollo de sus actividades, hacia la búsqueda de su crecimiento laboral, donde pueda convertirse en el activo más necesario en la organización. Es así como se requiere de líderes que permitan a sus seguidores a lograr cada uno de los objetivos planteados, proporcionando la libertad en sus acciones, aprovechando sus competencias (conocimientos, habilidades, destrezas, actitudes), estimulándolos 
para sercada día mejorpersona, un profesional más eficiente, facilitando el progreso, inspirándolos a lograr metas personales y organizacionales.

Por tanto, el líder resiliente debe tener la capacidad de gestionar el cambio, logrando fomentar en sus seguidores una visión proactiva, estratégica de su entorno organizacional, conociendo las necesidades de sus clientes internos y externos, así como innovación de los procesos que son claves para el éxito organizacional, sobre todo cuando se maneja la gestión del cambio.

\section{Cómo entender la resiliencia}

Hoy día, existe la tendencia de analizar y abordar el problema vinculado al talento humano de las organizaciones desde una perspectiva positiva, dejandoa unlado los problemas negativos de superación, de evitar responsabilidades y la poca relación con su entorno, por tanto, estudios vinculados a la psicología como los expuestos por Saavedra, Salas, Cornejo y Morales (2015), y Castañeda, Guevara, y Rodriguez (2005), demuestran que existen personas que mediante condiciones diversas, enfrentar con éxito sus adversidades, rompiendo las expectativas de fracaso que anteriormente se les otorgaban.

Según lo expresado, la calidad de vida de las personas, puede permite que se desarrollen nuevos mecanismos protectores que los convierten en seres resilientes, por ello, el término resiliencia, ha sido tomado de la metalurgia, donde se refiere a la capacidad de los metales en resistir los golpes y recuperar su estructura interna.

Dentro del contexto del desarrollo psicosocial del ser humano, el término de resiliencia implica la capacidad de recuperarse ante las adversidades a las cuales se enfrenta el individuo, logrando así una combinación de diversos factores que les permite superar los problemas, mejorando su autoestima, confianza, logrando generar una visión positiva ante lo vivido, en vez de enfatizar aquellos aspectos negativos que permitirían predecir el daño que podrían generar.

En consonancia con lo planteado, señala Rutter (1985), la resiliencia no debe ser entendida como la animada negación de las difíciles experiencias de la vida, dolores y cicatrices. Es más bien, la habilidad para seguir adelante a pesar de ello. Por tal motivo, el mismo no se debe ver como una condición negativa, es parte de un proceso que permite crear un nuevo individuo, el cual con afecto, positivismo, se responsabiliza de sus acciones y se enfrenta a experiencias que no imaginaba vivir. El autor citado señala "ser humano "en resiliencia", o "esfera de resiliencia" se concibe como un proceso dinámico de interacción entre factores o recursos personales y sociales que conforman una serie de posibilidades tanto de respuesta al conflicto como de potenciación de otras fuerzas personales y sociales con que las personas y comunidades de éxito enfrentan su realidad"

\section{Escenarios prospectivos}

Hablar de prospectiva se refiere al estudio del futuro, o de lo que pudiese pasar en el mañana, para comprenderlo y poder influir en él, basándose en la información del porvenir que conocemos en el presente, analizando que aspectos pudieran ser causa de diversos escenarios u opciones 
de hechos o sucesos venideros. Resulta una herramienta indispensable para la planeación a largo plazo, en busca de minimizar riesgos o en su defecto estar preparados ante posibles eventos que pudieran presentarse.

Según lo descrito por el Instituto de Prospectiva Estratégica de España (1999) éste define la prospectiva como una disciplina con visión global, sistémica, dinámica y abierta que explica los posibles futuros, no sólo por los datos del pasado sino fundamentalmente teniendo en cuenta las evoluciones futuras de las variables (cuantitativas y sobretodo cualitativas) así como los comportamientos de los actores implicados, de manera que reduce la incertidumbre, ilumina la acción presente y aporta mecanismos que conducen al futuro aceptable, conveniente o deseado.

A este respecto, Astigarraga (2016), define la prospectiva, como un proceso sistemático, participativo de construcción de una visión a largo plazo, para la toma de decisiones, así como las acciones a desarrollar. De acuerdo con lo planteado, Alonso (2011), sostiene que la prospectiva pretende aclarar los objetivos que buscan perseguirse, explorando los caminos viables, alternativos, especular y conjeturar sobre los posibles cambios, evaluando las consecuencias originadas por las acciones a emprender, y las resultantes de no actuar (acciones posibles, probables o deseables para el futuro), disminuyendo el factor sorpresa. La misma es a largo plazo, permitiendo imaginar un futuro diferente al presente.

Por otra parte, Mujica (2008), señala que la prospectiva, es un proceso intelectual a través del cual se representa tanto el futuro posible, es decir todo lo que puede ocurrir, como el futuro deseado (lo que nos gustaría), para la organización. Siendo una de las funciones más difíciles pero más atractiva de la prospectiva, el ser generadora de cambio.

Por su parte, Godet (2007), fundador del Círculo de Empresarios del futuro, y autor de diversas obras sobre prospectiva, plantea la compresión de futuro como si fuese un lienzo en blanco; opina que la prospectiva es una anticipación (pre activa y proactiva), para determinar las acciones presentes con la luz del futuro deseable y posible. Lo cual permite prepararse, adelantarse ante los cambios previstos e inclusive reaccionar para provocar los cambios deseados. El mencionado autor utiliza la lógica del triángulo griego, donde el color rojo de la anticipación sólo puede transformarse en el verde de la acción con el amarillo de la adaptación de los actores implicados.

La prospectiva se ha convertido en una herramienta de gran relevancia en la planeación estratégica de las organizaciones. En este orden de ideas, los escenarios constituyen una imagen del futuro probable, por medio de los cuales se examina detalladamente las opciones que se tienen ante determinada variable o decisión, permitiendo organizar los datos disponibles en el presente para ser proyectados a un futuro deseable.

El futuro tiene múltiples opciones, son precisamente los escenarios los que nos brindan diversas imágenes, así como descripciones de ese futuro, la manera en la que analizamos esos escenarios nos guiaran en las acciones 
para comprender como las decisiones que hoy tomamos serán las más asertivas y como podrán influir de manera positiva o negativamente en nuestro futuro. Los escenarios son una herramienta para saber que se debe hacer en el presente para obtener un resultado en el futuro.

Es importante revisar, que a nivel mundial la inclinación de los sistemas y métodos prospectivos han tenido su mayor aplicación en sectores de interés público o gubernamental, convirtiéndose en la herramienta principal de los planes y programas de los gobiernos en todas las instancias, siendo aplicadas en el desarrollo tecnológico, científico, social, defensa, entre otros.

En la medida en que se aplique o implementen métodos prospectivos en la gestión estratégica de las organizaciones, permitirá reducir los riesgos y el nivel de incertidumbre sobre la aplicación de los proyectos, dictando las pautas de las acciones y estrategias a seguir, contribuyendo a la toma de decisiones.

\section{Liderazgo resiliente prospectivo}

Los cambios constantes a nivel social, político, financiero, entre otros, ha creado la necesidad de adaptación continua en infinidad de disciplinas, desde el individuo como ente particular, hasta cualquier agrupación o conjunto de personas confines comunes, esto perfectamente reflejado en el plano organizacional, individuos relacionados y trabajando en el logro de un objetivo común.

A este respecto, las organizaciones no escapan de los cambios que suceden en su entorno, y en las vidas de los colaboradores que hacen vida activa dentro de su estructura, es por tal motivo, que a lo largo de la historia se han observado cambios significativos en los estilos gerenciales, tipos de liderazgos emergentes que buscan el beneficio organizacional, marcando cada día más la importancia del factor humano, y por ende, buscando mecanismos de motivación que permitan que cada integrante de la organización se sienta identificado y parte de ella.

En párrafos anteriores se han descrito, entre otras cosas, los beneficios que trae para la organización un liderazgo resiliente. Sin embargo, también se presentan líderes con mentalidad prospectiva, quienes buscan comprender el futuro para poder influirlo, para Machado (2008) la actitud de un líder prospectivo debe acercar el futuro para el análisis, proyectando acciones que permitan generar el futuro deseado, siendo esta una característica de un líder visionario.

Ahora bien, si un liderazgo prospectivo es capaz de fundar los cimientos de un porvenir deseado, un líder resiliente prospectivo pudiera preparar a sus seguidores para afrontar de mejor manera ese futuro, anticipándose a los factores que pudieran afectar el entorno organizacional. Esta es solo una idea, que da una puerta abierta a nuevas investigaciones, a nuevos conceptos en materia organizacional, en busca de teorías emergentes que contribuyan a un desarrollo cada día más humano dentro del mundo empresarial.

\section{Reflexiones finales}

Las organizaciones modernas les ha tocado vivir épocas muy cambiantes, cambios que surgen de manera tan emergente, constante y a un ritmo tan acelerado, nunca antes visto. 
En consecuencia la gerencia se ha visto en la imperiosa necesidad de realizar profundas modificaciones en su forma prácticas de gestión, ya no es suficiente trabajar bajo una planificación de estrategias conservadoras y tradicionales, se hace necesario evaluar todo lo que ocurre entre sus actores y entorno, desde el punto de vista social, económico, político y cultural.

En este orden de ideas, se plantea la necesidad de estrategias innovadoras, basadas en diversos escenarios posibles y probables, enfocar los esfuerzos en esas posibilidades futuribles, lograr contemplar estrategias aplicables de diferentes escenarios y que se proyecten los resultados esperados, la aplicación de diversos métodos prospectivos, permiten minimizar los riesgos y lograr aprovechar los recursos de las organizaciones,

Cualquier organización dedicada a un área en particular tiene la posibilidad de disponer de líderes resilientes que permitan ser una guía en aquel talento humano que lo requiera, convirtiéndose en modelo a seguir sobre la manera de enfrentarse a las adversidades, o a experiencias que pueden marcar la vida de cada persona, sin embargo, siendo capaces de sobrellevarlas, asumirlas, enfrentarlas de manera positiva.

En el marco de este contexto cuando se plantea una gestión prospectiva la gerencia puede enfrentar situaciones de resistencia ante escenarios futuros, en algunos casos por inseguridad, mal manejo de las variables de estudio, temor al riesgo o por no conocer los riesgos ni las incidencias de que pudieran llegar a tener las decisiones tomadas bajo este tipo de premisas. Lo cierto, es que mediante un buen y sustentado análisis se minimizan los riesgos y se puede tener una visión bastante acertada de esa realidad futurible.

Es en este orden de ideas que el liderazgo resiliente cobra su protagonismo, con esa capacidad de reponerse rápidamente y enfrentar los constantes cambios que pudieran presentarse. Es por ello, que el estudio del liderazgo y la resiliencia ha sido analizado desde diversas perspectivas y orientaciones, sin embargo, cada día se demuestra la necesidad de su aplicabilidad en todos los contextos, logrando que los resultados obtenidos, sirvan de base para nuevos estudios y experiencias, generando mayores teorías al conocimiento científicos que sean soluciones viables a los problemas de la sociedad y de cada uno de los miembros que la conforman.

\section{Referencias bibliográficas}

Alianza Revista. (2008). Hacia un liderazgo prospectivo aplicado a la organización. Documento en línea. Disponible en https://docplayer.es/10251729-Hacia-unliderazgo-prospectivo-en-la-organizacion. html

Alonso, A. (2011). Prospectiva y Planeación. Analítica Consultores SA de CV,México D.F. Ponencia presentada en el Seminario Internacional "Experiencias de Planeación en América Latina y el Caribe", Centro Nacional de Planeamiento Estratégico (CEPLAN), Lima, Perú, noviembre 3-4, 2011.

Astigarraga, E. (2016). Prospectiva estratégica: orígenes, conceptos clave e introducción a 
su práctica. Icap-revista centroamericana de administración pública. V.71. Pág. 1329.

Castañeda, P. \& Guevara, A. (2005). Estudio de casos sobre factores resilientes en menores ubicados en hogares sustitutos. Pontificia Universidad Javeriana. Facultad de Psicología. Bogotá D.C.: Colombia.

Godet, M. (2007). Prospectiva Estratégica: problemas y métodos. San Sebastian, España: PROSPEKTIKER.

Instituto de Prospectiva Estratégica de España. (1999). Documento en línea: José Miguel Echarri. Disponible en www. prospecti.es/ipeframe.htm

Medina, L. (2015). Liderazgo y Resiliencia. Una revisión de la literatura.Trabajo especial de grado. Universidad del Rosario. Bogotá, Colombia.

Mujica, F. (2008). Forecasting y Prospectiva dos alternativas complementarias para adelantarnos al futuro. Bogotá: Universidad Externado de Colombia.

Richardson, T. (2014). Resiliencia: Estado actual y Enfoques. Madrid. España: Editorial Norma.

Robbins, S. (2015). Comportamiento organizacional. México, D.F.: Editorial Prentince Hall.

Kothiarenco, M.; Álvarez, C. \& Cáceres, I. (1996). compiladores. Resiliencia: Construyendo en adversidad. Santiago (Chile): CEANIM; pp. $51-64$.

Rutter, M. (1993). La "Resiliencia":
Consideraciones Conceptuales. Journal of Adolescent Health, 14(8), 690-696.

Saavedra, E.; Salas, G.; Cornejo, C. \& Morales, P. (2015). Resiliencia y calidad de vida. La Psicología Educacional en diálogo con otras disciplinas. Primera Edición: Agosto 2015. ISBN, 978-956.

Meneghel, I.; Salanova, M.; \& Martínez, I. (2013). El camino de la Resiliencia Organizacional-Una revisión teórica. Aloma: Revista de Psicologia, Ciències de l'Educació i de l'Esport, 31(2).

Trujillo, J.; López, J. \& Lorenzo, M. (2011). Análisis y descripción de las percepciones del liderazgo resiliente y liderazgo distribuido en torno al ejercicio directivo (2.0) como posibilidad para aprehender y transformar las instituciones educativas. REICE: Revista Iberoamericana sobre Calidad, Eficacia y Cambio en Educación, 9(3), 13-29. 